\title{
Simetría-asimetría cognitiva y afinidad socioafectiva en la comprensión colaborativa de tablas de frecuencias
}

\author{
Cognitive symmetry-asymmetry and socio-affective affinity in the collaborative \\ comprehension of frequency tables
}

\author{
Mariano Castellaro ${ }^{\mathrm{a}, \mathrm{c},}$, Néstor Roselli ${ }^{\mathrm{b}, \mathrm{c}}$ \\ aUniversidad Nacional de Rosario, Argentina

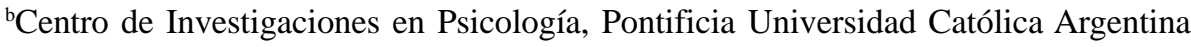 \\ cConsejo Nacional de Investigaciones Científicas y Técnicas, Argentina
}

\section{Resumen}

Objetivos: se analizaron los efectos de la simetría-asimetría de competencia y de la afinidad socioafectiva sobre la interacción colaborativa, en la comprensión de una tabla de frecuencias. Método: se distribuyeron en díadas simétricas y asimétricas, según igualdad-diferencia entre las competencias específicas de sus integrantes, y, a su vez, con y sin afinidad socioafectiva recíproca. Participaron 90 estudiantes (45 díadas) de sexto y séptimo grado de escolaridad primaria (edad, $M=12.3, D E=.5$ ), seleccionados de manera no probabilística. Cada díada resolvió 12 ítems de elección múltiple; cada uno de estos evaluaba un aspecto de la comprensión de la tabla. La interacción colaborativa fue analizada desde un sistema de categorías de tres niveles jerárquicos. Resultados: sugirieron una influencia de la afinidad socioafectiva al interior de la asimetría cognitiva, en tanto la presencia de la primera se asoció con un menor desequilibrio de los aportes entre los compañeros, relativos a la resolución de la tarea. En cambio, en la simetría de competencia, la afinidad socioafectiva se asoció con mínimas variaciones. Conclusiones: los hallazgos se discuten desde una perspectiva situada o contextualizante del hecho sociocognitivo, que pretende superar la concepción clásica epistémica.

Palabras clave: colaboración entre pares, simetría cognitiva, asimetría cognitiva, afinidad socioafectiva, tablas de frecuencias.

\section{Para citar este artículo:}

Castellaro, M., \& Roselli, N. (2019). Simetría-asimetría cognitiva y afinidad socioafectiva en la comprensión colaborativa de tablas de frecuencias. Liberabit, 25(2), 213-231. https://doi.org/10.24265/ liberabit.2019.v25n2.06

\begin{abstract}
Objectives: The effects of competence symmetry-asymmetry and socio-affective affinity on the collaborative interaction to understand a frequency table were analyzed. Method: The participants were distributed in symmetric and asymmetric dyads taking into account the similarities and differences of their specific competences, and if they had or not reciprocal socio-affective affinity. Ninety (90) students (45 dyads) attending the sixth and seventh grades of primary school (age, $M=12.3, S D=.5$ ), who were screened using a non-probability method, participated in the study. Each dyad answered 12 multiple-choice questions, each of which evaluated one aspect of the table comprehension. The collaborative interaction between the participants was analyzed from a three-hierarchical-level category system. Results: Socioaffective affinity has influence on cognitive asymmetry. It is associated with a lower imbalance of peers' contributions related to the resolution of the task. In contrast, in competition symmetry, socio-affective affinity was associated with slightest variations. Conclusions: The findings are discussed in terms of a situated or contextualizing perspective of the socio-cognitive event which aims to overcome the traditional epistemic conception.
\end{abstract}

Keywords: peer collaboration, cognitive symmetry, cognitive asymmetry, socio-affective affinity, frequency tables.

Este es un artículo Open Access bajo la licencia Creative Commons Atribución-NoComercial-CompartirIgual 4.0

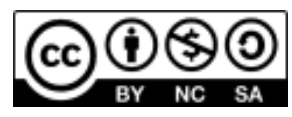




\section{Introducción}

El estudio de la relación entre la interacción colaborativa entre pares y sus efectos cognitivos cuenta con una amplia literatura de base. Parte de esas investigaciones han analizado diversos aspectos intervinientes en dicha relación, entre los cuales se destaca el grado de igualdad o desigualdad entre las competencias específicas de los compañeros de trabajo. El carácter «específico» de dicha competencia refiere a las particularidades cognitivas que plantea la tarea puntual en la cual es analizada la interacción sociocognitiva y que hacen al contexto de actividad (Psaltis, Duveen, \& Perret-Clermont, 2009).

A partir de esta estrecha conexión entre cognición, interacción y tarea, es esperable que la similitud o diferencia entre las habilidades de los compañeros se traduzca en formas sociocognitivas diferentes y en distintos grados de progreso cognitivo (Asterhan, Schwarz, \& Cohen-Eliyahu, 2014; Buchs \& Butera, 2009; Buchs, Darnon, Quiamzade, Mugny, \& Butera, 2008; Denessen, Veenman, Dobbelsteen, \& Schilt, 2008; Schmitz \& Winskel, 2008). Se utilizará el término simetría-asimetría de competencia para aludir a esta variable. Algunas de estas investigaciones revalorizan la asimetría (moderada) de competencia como fuente de cambio o progreso cognitivo, sobre todo para el individuo menos hábil (Asterhan et al., 2014; Denessen et al., 2008). Otros trabajos, mayormente emparentados con la psicología genética piagetiana, enfatizan el intercambio entre individuos con similares niveles de competencia específica y diferentes perspectivas individuales (Peralta \& Roselli, 2017).

Con todo, han surgido nuevas investigaciones al respecto que implican un cambio en el enfoque conceptual de la cuestión. Este consiste en el pasaje desde una concepción epistémica o cognitivista hacia una concepción psicosocial (Castorina, 2018; Staerklé \& Butera, 2017), que implica la consideración de aspectos psicosociales involucrados en la interacción (Quiamzade, Mugny, \& Butera, 2014), superando una visión abstracta del proceso colaborativo, equiparable a una suerte de pensamiento en red o de multiagentes. En esta nueva perspectiva, la coordinación con otros implicaría un pensamiento colectivo situado, mediatizado por roles asignados o atribuciones representacionales recíprocas de los individuos que interactúan, permitiendo profundizar la comprensión de dicho fenómeno (Psaltis \& Zapiti, 2014). Este nuevo enfoque mantiene el interés por el estudio de la simetría-asimetría de competencia cognitiva, pero lo vincula con otras formas de simetría-asimetría asociadas con aspectos psicosociales, no cognitivos en sentido estricto. Se trata de entender a la igualdad o diferencia interpersonal no solo en términos cognitivos genéricos abstractos, sino situados, incorporando distintas variables psicosociales que también definen formas de simetría o asimetría (Psaltis et al., 2009).

Un claro exponente son las investigaciones que analizan el conflicto sociocognitivo entre pares en combinación con la igualdad o diferencia de género en díadas. Esta línea de trabajo fue iniciada por Leman y Duveen (1999), quienes observaron díadas de individuos de nueve años en una tarea colaborativa de razonamiento moral (Piaget, 1984). Las díadas estaban integradas por un participante autónomo y uno heterónomo (en el sentido de la psicología genética piagetiana) ${ }^{1}$, aunque variaban en cuanto al género: niña-niña, niño-niño, niña-niño. Los resultados sugirieron que el comportamiento del individuo autónomo es afectado por el género: las niñas

\footnotetext{
Desde una perspectiva piagetiana ortodoxa, un pasaje central dentro del desarrollo moral refiere a la transición heteronomía-autonomía, la cual constituye el emergente de una transformación de las estructuras del pensamiento desde una lógica preoperatoria hacia una lógica operatoria. Simplificando, una moral heterónoma es aquella supeditada a una autoridad exterior, que generalmente es la del adulto, y que está basada en una relación de imposición o unilateralidad. En cambio, una moral autónoma (superadora de la anterior) está basada en la mutualidad, reciprocidad y cooperación; por tanto, las reglas no dependen de una autoridad que las impone sino que se basan en una idea de justicia social, en la cual se integran el propio punto de vista del individuo y el de los demás (Piaget, 1995).
} 
autónomas, en comparación con los varones autónomos, dedican mayor tiempo y un amplio rango de argumentos hacia su compañera/o heterónoma/o. También son diferentes los patrones conversacionales al inicio del diálogo: los niños autónomos tienden a imponer su perspectiva, dando al compañero heterónomo escasa oportunidad de cuestionar o desafiar su opinión. En cambio, las niñas autónomas frecuentemente inician la conversación con preguntas destinadas a conocer el fundamento de la opinión del individuo heterónomo y tratar de construir una solución que integre ambas opiniones. Estudios posteriores basados en el mismo esquema metodológico se aplicaron a tareas de construcción de nociones lógicas de conservación (Psaltis \& Duveen, 2007) y espacial (Psaltis, 2011; Zapiti \& Psaltis, 2012, 2019) en niños de menor edad (seis y siete años). Los resultados arrojaron conclusiones similares a los estudios previos.

Otra variable psicosocial de relevancia es la amistad o afinidad socioafectiva entre los compañeros de trabajo. No se registran antecedentes que analicen la influencia de la simetría-asimetría de competencia cognitiva y de afinidad socioafectiva sobre la interacción colaborativa entre pares. Sin embargo, hay trabajos referidos a los efectos de la amistad sobre la interacción, sin incluir la simetría-asimetría de competencia cognitiva, en tareas colaborativas libres o expresivas. McDonald, Miell y Mitchell (2002) trabajaron con díadas de ocho y once-doce años, en tareas de composición musical. Según estas investigaciones, la afinidad entre los compañeros favorece un mayor volumen y calidad en la comunicación verbal, comparado con díadas integradas por no afines. Esta variable también fue estudiada en combinación con el género. Strough, Swenson y Cheng (2001) trabajaron con díadas de 6. ${ }^{\circ}$ grado que debían realizar una tarea de escritura colaborativa de historias. Los resultados reafirmaron que la afinidad favorece el compromiso mutuo y la coordinación intersubjetiva en la actividad, así como una representación más positiva de la propia experiencia colaborativa.
En función de lo anterior, se proponen dos aportes fundamentales. Por un lado, se pretende estudiar la interacción colaborativa entre pares desde una perspectiva psicosocial, analizando la simetríaasimetría de competencia cognitiva en combinación con la afinidad socioafectiva. Pero, además, se propone hacerlo en un tipo de tarea poco considerado en estudios sobre colaboración entre pares: la comprensión de tablas de frecuencias. Así, el estudio apunta a complementar dos líneas de investigación ya consolidadas, aunque poco conectadas entre sí: sobre interacción colaborativa entre pares y sobre sistemas de representación externa (Pérez, Martí, \& Pozo, 2010).

Un enfoque interaccionista del conocimiento asume que este se produce necesariamente en torno a una mediación representacional externa (soporte objetivo o material), lo cual puede referir a diferentes sistemas (Andersen, Scheuer, Pérez, \& Teubal, 2017; Pérez et al., 2010; Pozo, 2017). En ese sentido, el concepto de sistema externo de representación se asocia con aquellos dispositivos culturales que extienden o amplían (en tanto soporte) las funciones mentales, incluso llegando a constituirse como elemento intrínseco de dichos procesos. Estos sistemas implican tanto al lenguaje escrito como a otros sistemas externos permanentes, cada uno definido por características particulares y niveles propios de complejidad, por ejemplo, la notación numérica (Martí, Scheuer, Cavalcante, Trench, \& Brizuela, 2016; Rodríguez, Martí, \& Salsa, 2018), los gráficos (Pérez, Postigo, \& Marín, 2018; Postigo \& LópezManjón, 2015; Salsa \& Gariboldi, 2017; Salsa \& Vivaldi, 2017) o los mapas (Maita, Mareovich, \& Peralta, 2014), entre otros.

Dentro de este amplio conjunto, las tablas de frecuencias constituyen un sistema particular, donde la representación externa u objetiva del conocimiento se hace a través de un formato espacial bidimensional que contiene cantidades numéricas (Gabucio, Martí, Enfedaque, Gilabert, \& Konstantinidou, 2010; Martí, García-Milá, Gabucio, \& Konstantinidou, 2011). En 
la educación formal, los procesos de instrucción para el manejo de este tipo de sistemas comienzan, en términos aproximados, hacia finales del ciclo primario, equivalente a sexto y séptimo grado en el sistema de educación formal de Argentina.

Gabucio et al. (2010) estudiaron el nivel de comprensión individual de tablas de frecuencias, en estudiantes catalanes de $5 .^{\circ}$ y $6 .^{\circ}$ de primaria, y de $1 .^{\circ}$ y $2 .^{\circ}$ de ESO. Una tabla de doble entrada resumía los datos de la distribución de frecuencias de un supuesto grupo de alumnos pertenecientes a un curso escolar y combinaba dos variables: el peso (en kilogramos) distribuido en cuatro rangos (menos de 25, de 25 a 34, de 35 a 44 y más de 44) y el sexo (niñas y niños). También incluía los totales del eje vertical, aunque no los del eje horizontal. Cada alumno leía individualmente la tabla y respondía una serie de 12 preguntas presentes en un cuestionario que indagaban el nivel de comprensión relativo a la estructura y al contenido de dicho sistema representacional.

Los autores identificaron cuatro niveles progresivos de complejidad en la comprensión del sistema representacional: (1) lectura directa de los datos de la tabla, (2) comprensión de la estructura tabular, (3) inferencias particulares a partir de los datos de la tabla, y (4) comprensión global del contenido de la tabla. El primero refiere al reconocimiento, localización y extracción directa de datos explícitos de la tabla. El segundo remite al entendimiento de lo que implica la estructura y ordenamiento de una tabla. Si bien este último comparte con el primero su vinculación con los aspectos descriptivos o explícitos de la tabla, al mismo tiempo implica un cierto desprendimiento de los aspectos inmediatamente perceptibles y una identificación de las reglas internas de dicho sistema. El tercer nivel de comprensión refiere a la identificación de relaciones implícitas entre los datos de la tabla, a partir de la integración e interpretación de estos. Por último, el cuarto nivel, de comprensión más profunda, remite a un proceso de inferencia o interpolación global, es decir, que permite la generación de nuevas ideas y predicciones a partir de los datos de la tabla. En suma, mientras los niveles 1 y 2, aún con sus diferencias, refieren a «leer los datos», el nivel 3 se vincula con «leer entre los datos» y el nivel 4 con «leer más allá de los datos».

Si bien se ha retomado la tarea utilizada por Gabucio et al. (2010), el análisis versa sobre la interacción colaborativa de díadas, es decir, realizada entre dos compañeros. La interacción colaborativa es analizada puntualmente en términos de simetríaasimetría del intercambio, es decir, el equilibrio entre los diferentes tipos de aportes verbales de los compañeros de trabajo durante la realización de la tarea (Castellaro \& Roselli, 2018a, 2018b).

En síntesis, el objetivo del estudio es analizar los efectos de dos variables: la simetría-asimetría de competencia cognitiva relativa al nivel de comprensión de la tabla de frecuencias (simetría: dos estudiantes de competencia básica; asimetría: un estudiante de competencia básica y uno de competencia avanzada), y la afinidad socioafectiva recíproca entre los estudiantes (presencia y ausencia). Ambas variables son estudiadas según su influencia sobre la interacción colaborativa, considerada en términos de equilibrio o desequilibrio entre los aportes individuales de los compañeros. Esto se analizó en díadas de $6 .^{\circ}$ y $7 .^{\circ}$ grado de primaria. Los objetivos específicos son tres: (a) analizar la influencia de la simetría-asimetría de competencia sobre la interacción colaborativa, (b) analizar la influencia de la afinidad socioafectiva sobre la interacción colaborativa, y (c) analizar el efecto combinado de la simetría-asimetría de competencia y de la afinidad socioafectiva sobre la interacción colaborativa.

\section{Método}

\section{Diseño}

El diseño apuntó a analizar las variaciones generadas por las dos variables referidas (competencia cognitiva específica y afinidad socioafectiva), respecto a la interacción colaborativa, considerada en términos 
de la distribución y equilibrio entre los aportes verbales de los compañeros de la díada. La primera variable independiente (simetría-asimetría de competencia cognitiva de la díada) fue definida por la igualdad o diferencia entre los niveles de comprensión de la tabla por parte de los compañeros de la díada. Así, se distinguieron dos tipos de díada: ambos participantes con igual nivel de competencia (díadas simétricas) y ambos participantes con distinto nivel de competencia (díadas asimétricas). En el caso puntual de la simetría, esta se circunscribió a los casos de baja competencia, ya que eran los más frecuentes y, por otra parte, era de suponer que en estos casos la afinidad socioafectiva resultaría más condicionante de la interacción como producto de una compensación del menor desarrollo de dicha competencia específica.

La segunda variable independiente, afinidad socioafectiva de la díada, fue definida por la presencia o ausencia de una elección recíproca entre los miembros de la díada para realizar la tarea colaborativa. Se estudiaron dos categorías: díadas con afinidad socioafectiva recíproca, cuando ambos participantes se eligieron mutuamente para trabajar en conjunto, y díadas sin afinidad socioafectiva recíproca, cuando ambos participantes no se eligieron mutuamente para trabajar en conjunto. Se omitió una situación intermedia o parcial de afinidad socioafectiva, cuando solo uno de los individuos elige trabajar con un determinado compañero, mientras este no. Esta decisión se fundamentó en el interés por estudiar las dos formas extremas de reciprocidad electiva.

\section{Participantes}

Noventa estudiantes (45 díadas) de $6 .^{\circ}$ y $7 .^{\circ}$ grado de escolaridad primaria (edad en años: $M=12.3 ; D E$ $=.5$ ) (ciudad y país omitidos por anonimato de los autores), distribuidos equitativamente según sexo y curso. Estos residían en sectores urbanizados, en una de las ciudades con mayor índice poblacional del país y ubicada en la zona central del mismo. En su gran mayoría, todos pertenecían a un nivel socioeconómico medio. Los casos fueron seleccionados intencionalmente en función de la integración pautada de las díadas según las distintas condiciones de comparación. Cada condición estuvo conformada por los siguientes participantes: simetría con afinidad ( $n$ $=10)$, simetría sin afinidad $(n=13)$, asimetría con afinidad $(n=11)$, y asimetría sin afinidad $(n=11)$.

\section{Materiales}

La tarea principal consistió en analizar una tabla de frecuencias y responder 12 preguntas referidas a la comprensión del sistema representacional (ver en Anexo la tabla y ejemplos de preguntas). Cada pregunta contaba con cuatro opciones de respuesta y solo una de estas era elegible. Cada una de las 12 preguntas remitía a uno de los cuatro niveles de comprensión de la tabla: lectura directa de los datos de la tabla (ítems 4, 5 y 10); comprensión de la estructura tabular (ítems 1, 2 y 3); inferencia a partir de los datos de la tabla (ítems 7, 8, 9 y 11); y comprensión global de la tabla (ítems 6 y 12).

Por otra parte, para la identificación de elecciones socioafectivas recíprocas y no recíprocas entre los individuos se utilizó un criterio basado en Strough, Berg y Meegan (2001) y Swenson y Strough (2008). El mismo consistió en la utilización de una grilla simple con espacios vacíos, en los cuales cada estudiante proponía compañeros de clase de cualquier género con quienes más le gustaría trabajar en equipo durante una actividad escolar. Aquí, cada participante propuso 10 compañeros de clase, ordenados en un rango de 1 a 10 (de mayor a menor afinidad). Este proceder se fundamenta en el próximo apartado.

\section{Procedimiento}

Se obtuvieron los consentimientos informados de la institución escolar, de los padres y de los propios participantes. No participaron del estudio quiénes no contaron con alguna de estas autorizaciones. En todo momento, se respetaron las legislaciones y regulaciones nacionales e internacionales vigentes sobre investigaciones sociales. Asimismo, la confidencialidad y anonimato de toda la información fue mantenida 
acorde a lo establecido por dicha legislación. Esto significa que los datos obtenidos solo fueron utilizados por los investigadores en el contexto de este estudio, no pudiendo ser empleados para finalidades distintas o incompatibles con aquellas que motivaron su obtención.

Conformación de las díadas. Al interior de cada curso, cada estudiante realizó individualmente la tarea de comprensión de la tabla (12 ítems o preguntas de opción múltiple). En base a esto, se diferenciaron participantes con dos niveles globales de comprensión de la tabla: básico y avanzado. El nivel general básico correspondía a aquellos estudiantes que alcanzaban, como máximo, un nivel específico 1 o 2 de comprensión de la tabla; en cambio, el nivel general avanzado correspondía a aquellos estudiantes que alcanzaban un nivel específico 3 o 4 de comprensión de tabla. Ahora bien, para determinar como válido ese nivel específico máximo alcanzado se tomó un criterio gradual correlativo, es decir, solo se aceptaba como válido si el estudiante también demostraba respuestas correctas en los ítems correspondientes a los niveles específicos menos complejos. Esto se realizó con el fin de controlar el efecto de respuestas de tanteo o arbitrarias. En la Tabla 1 se describen los criterios correlativos utilizados para determinar el nivel general de comprensión de la tabla.

\section{Tabla 1}

Criterios para determinar el nivel general de comprensión de la tabla

\begin{tabular}{|c|c|c|c|c|c|c|}
\hline & & & \multicolumn{4}{|c|}{ Criterios correlativos de comprensión (comp.) } \\
\hline & & & $\begin{array}{c}\text { Hasta } 3 \text { ítems } \\
\text { correctos de } \\
\text { lectura } \\
\text { directa }\left(^{*}\right)\end{array}$ & $\begin{array}{l}2-3 \text { ítems } \\
\text { correctos de } \\
\text { comp. estructura } \\
\text { tabular }\end{array}$ & $\begin{array}{c}\text { 2-4 ítems } \\
\text { correctos de } \\
\text { inferencia de } \\
\text { datos }\end{array}$ & $\begin{array}{l}\text { 1-2 ítems } \\
\text { correctos de } \\
\text { inferencia } \\
\text { global }\end{array}$ \\
\hline \multirow{4}{*}{ Nivel general de comp. } & \multirow[t]{2}{*}{ Básico } & Nivel 1 & $X$ & & & \\
\hline & & Nivel 2 & $X$ & $X$ & & \\
\hline & \multirow[t]{2}{*}{ Avanzado } & Nivel 3 & $\mathrm{X}$ & $\mathrm{X}$ & $\mathrm{X}$ & \\
\hline & & Nivel 4 & $X$ & $\mathrm{X}$ & $X$ & $X$ \\
\hline
\end{tabular}

Al finalizar lo anterior, también de manera individual, cada estudiante completó la grilla sociométrica, es decir que propuso 10 compañeros o compañeras del curso, con quienes más les gustaría trabajar en una actividad colaborativa escolar. El propósito era detectar elecciones socioafectivas recíprocas y no recíprocas entre compañeros de una misma clase. La afinidad socioafectiva entre dos participantes existía cuando había reciprocidad electiva dentro de las tres primeras elecciones. En cambio, la ausencia total de afinidad socioafectiva entre dos participantes suponía la ausencia total de reciprocidad dentro de las 10 elecciones solicitadas. No se tuvieron en cuenta elecciones parciales o no recíprocas.

A partir de ambas evaluaciones (nivel de comprensión individual de la tabla y afinidad socioafectiva) se fueron conformando intencionalmente las díadas correspondientes a las cuatro condiciones de estudio. Las díadas consideradas 
como simétricas estuvieron integradas por dos estudiantes de competencia básica (nivel 1 o 2). Por su parte, las díadas consideradas como asimétricas estuvieron conformadas por un participante de competencia básica (nivel 1 o 2) con otro de competencia avanzada (nivel 3 o 4), con una diferencia mínima entre ambos de los dos niveles (por ejemplo, un participante de nivel 1 con uno de nivel 3 o 4, o uno de nivel 2 con otro de nivel 4). A su vez, las díadas simétricas y asimétricas estuvieron distribuidas equitativamente en función de la presencia y ausencia de afinidad socioafectiva entre sus integrantes.

Fase colaborativa. Entre las dos y tres semanas posteriores, cada díada resolvió la misma tarea realizada que en la fase individual, solo que en esta instancia, la consigna era claramente colaborativa: ambos compañeros debían resolver la actividad de manera conjunta, tratando de discutir acerca de la solución correcta de cada pregunta y tratando de lograr una decisión compartida. No se estableció un tiempo límite de realización. La actividad tuvo lugar en el horario habitual de cursado, en un espacio diferente a la sala de clases. La interacción completa fue audiograbada y transcripta.

\section{Categorías de análisis de la interacción colaborativa}

La codificación de la interacción colaborativa partió de la distinción entre unidad dialógica (o turno conversacional) y unidad cognitiva (Roselli, 2016). Una unidad dialógica consiste en un segmento comunicacional verbal a cargo de uno de los participantes que finaliza por la intervención posterior de su compañero o de manera espontánea. En cambio, la noción de unidad cognitiva refiere al elemento cognitivo más simple en el que se puede dividir un segmento comunicacional, el cual reconoce diferentes categorías posibles. De esta manera, puede ocurrir que una unidad dialógica esté integrada por una o varias unidades cognitivas (simple o compuesta, respectivamente). En el presente análisis categorial se tomó como unidad de referencia a cada unidad cognitiva (más allá de que esta haya coincidido con un turno conversacional completo o solo haya constituido una parte del mismo).

El sistema categorial de las unidades cognitivas utilizado se basó en una adaptación de sistemas previos (Castellaro \& Roselli, 2018b; Roselli, 2011; Roselli, Dominino, \& Peralta, 2010). El mismo estuvo constituido por tres niveles categoriales inclusivos entre sí (de mayor a menor generalidad), cada uno de estos integrado por códigos mutuamente excluyentes y exhaustivos.

\section{Nivel 1}

- Aporte de resolución de la tarea: aporte de conocimiento (e.g., correcto o incorrecto; nuevo o ya emitido durante la interacción) orientado directamente a la solución del problema.

- Aporte de organización de la tarea vinculado con su resolución: si bien no constituye un aporte de conocimiento específico, predispone o constituyen un preludio con respecto al primero.

- Aporte de organización de la tarea no vinculado a su resolución: remite a aspectos organizativos secundarios de la tarea, sin constituir un aporte de conocimiento específico ni tampoco predisponer a este.

\section{Nivel 2a (solo para aportes de resolución de la tarea):}

- Afirmaciones

- Preguntas

- Respuestas (solo se consideró como respuesta a la unidad cognitiva inmediatamente posterior a una pregunta previa que guardaba una relación de sentido con esta). 


\section{Nivel 2b (solo para aportes de organización de la} tarea vinculados con su resolución):

- Lectura verbal de la consigna o de las opciones de respuesta.

- Lectura verbal exploratoria de elementos de la tabla: exploración y observación superficial de aspectos específicos de la tabla (celdas, totales, categorías, etc.). Este código debe diferenciarse de las unidades cognitivas en las que se apela a elementos de la tabla para fundamentar una propuesta de solución del problema (afirmación fundamentada de nivel 3 ), por ejemplo, «(observando los datos en celdas de la tabla)... sería 25...25 más 16... un 0 y un 1, serían...eh...45, 40, 40, 48...».

- Orientación: unidad dirigida al compañero, a través de la cual se le sugiere la realización de una acción determinada que predispone a la construcción de la solución de la tarea, por ejemplo, «esto es así, mira» (previo al desarrollo de una idea).

Nivel 3 (solo para aportes de resolución de la tarea):

- Afirmación fundamentada de aporte a la resolución de la tarea: dirigida a la solución de la tarea, correcta o incorrecta, acompañada por una justificación o fundamentación explícita en la cual se basa, por ejemplo, «la respuesta es 6 porque dice niñas (en la consigna) y acá (señala la celda correspondiente) hay 6. 16 es la suma de los niños más las niñas» (en ítem 5).

- Afirmación no fundamentada de aporte a la resolución de la tarea: dirigida a la solución de la tarea (correcta o incorrecta) de manera directa, es decir, sin un intento de justificación o fundamentación explícita de la misma, por ejemplo, «para mí es niños y niñas, la a (opción a del ítem)».
- Afirmación reactiva (feedback) vinculada a la resolución de la tarea: intervención breve que retroalimenta un aporte previo de resolución de la tarea, propio o del compañero, sin aportar explícitamente una propuesta de solución (fundamentada o no fundamentada), por ejemplo, ante el aporte de un compañero: «la respuesta correcta es la b»; el estudiante responde simplemente: «estoy de acuerdo».

- Pregunta de requerimiento de opinión o acuerdo: dirigida al compañero con el propósito de consultar su grado de acuerdo con respecto a una idea propia o consensuar una propuesta de solución de la tarea, por ejemplo, «¿te parece poner la 2 (opción de respuesta)?».

- Pregunta de cuestionamiento: dirigida al compañero con el propósito para evaluar o a revisar una solución del problema propuesta por este. Frente a la afirmación «acá me parece que va el 5 (opción de respuesta)», el individuo responde: «¿el 5?». Luego de eso, el primer participante dice «sí».

- Pregunta de demostración: intervención dirigida al compañero para exponerle o presentarle una idea o solución de problema, por ejemplo, "¿ves que acá se va formando así?, ¿ves?» (mientras presenta una idea al compañero).

- Respuesta fundamentada de aporte a la resolución de la tarea: respuesta a una pregunta inmediatamente anterior, en la cual se intenta justificar o fundamentar, de manera correcta o incorrecta, una idea relativa a la solución de la tarea.

- Respuesta no fundamentada de aporte a la resolución de la tarea: respuesta a una pregunta inmediatamente anterior, en la cual se expone una idea relativa a la solución de la tarea de manera directa, es decir, sin un 
intento de justificación o fundamentación (puede ser correcta o incorrecta).

Como se mencionó líneas arriba, la interacción colaborativa fue analizada en términos de la distribución (más o menos equilibrada) de los aportes individuales de los compañeros de trabajo. Esto se determinó en relación a cada categoría de unidad cognitiva (considerada de manera independiente de las demás), dividiendo entre sí el número de unidades cognitivas (de esa categoría) aportados por cada participante. En esta división, el valor del numerador correspondió al individuo con menor aporte de unidades cognitivas, de manera de arrojar un valor entre 0 (desequilibrio máximo posible) y 1 (equilibrio máximo posible). Además, al momento de analizar cada categoría específica, si una díada registraba ausencia total de la misma (ninguno de los dos participantes la había emitido), directamente se la consideraba como caso perdido.

El análisis tuvo tres momentos, en función de los tres objetivos específicos planteados en la introducción. Así, cada código de interacción fue analizado en función de: (a) simetría-asimetría de competencia, (b) afinidad socioafectiva, y (c) simetríaasimetría de competencia y afinidad socioafectiva. Podría afirmarse que los pasos (a) y (b) fueron preliminares, mientras que el (c) fue el objetivo central del estudio, puesto que la interacción es analizada en función de la combinación entre simetría-asimetría de competencia y afinidad socioafectiva.

\section{Resultados}

\section{(a) Análisis de la interacción colaborativa en función de la simetría-asimetría de competencia de la díada}

La exploración inicial de los datos indicó que algunas categorías de interacción registraron frecuencias nulas o alta homogeneidad en gran parte de muestra. Esto sugirió de antemano que las mismas no estarían asociadas significativamente con las variables independientes del estudio (simetría-asimetría de competencia y afinidad socioafectiva). Dichas categorías fueron las preguntas de demostración y de cuestionamiento, y las respuestas fundamentadas dirigidas a la resolución de la tarea (todas de nivel 3). Por tanto, estas tres categorías fueron omitidas de los posteriores análisis y tablas. Por otra parte, considerando la libre distribución de los datos, el tamaño de muestra y la ausencia de igualdad entre varianzas, se utilizaron pruebas no paramétricas para analizar la significación de los valores descriptivos.

La Tabla 2 presenta los valores descriptivos relativos a la distribución de cada categoría de interacción en las dos condiciones de simetría-asimetría de competencia.

En relación a los aportes de resolución de la tarea (nivel 1), se observó un mayor equilibrio de la interacción en las díadas simétricas en comparación con las asimétricas. Esta tendencia fue más marcada en sus subcategorías de nivel 2 y 3 . Tal fue el caso de las preguntas dirigidas a la resolución de la tarea de nivel $2(U=121.00 ; p<.05)$ y las afirmaciones reactivas vinculadas a la tarea de nivel $3(U=131.00$; $p<.01)$. En las afirmaciones fundamentadas y no fundamentadas de resolución de la tarea (de nivel 3) también se observó una tendencia similar, aunque estas no llegaron a registrar diferencias significativas.

Por su parte, las unidades organizativas vinculadas a la resolución de la tarea (nivel 1) también se asociaron con intercambios más equitativos en las díadas simétricas $(U=135.50 ; p<.01)$. Esto también se dio particularmente en las unidades destinadas a la lectura verbal de la consigna o de las opciones de respuesta de nivel $2(U=134.50 ; p<.01)$. Por último, las unidades organizativas no vinculadas a la resolución de la tarea (nivel 1), también se distribuyeron más equitativamente cuando los compañeros de trabajo contaban con similares niveles de competencia específica. 
Tabla 2

Valores descriptivos de cada categoría de la interacción, en función de la simetría-asimetría de competencia de la díada

\begin{tabular}{|c|c|c|c|c|c|c|}
\hline & \multicolumn{6}{|c|}{ Simetría-asimetría de competencia } \\
\hline & \multicolumn{3}{|c|}{ Simetría $(n=23)$} & \multicolumn{3}{|c|}{ Asimetría $(n=22)$} \\
\hline & $M d n$ & $M$ & $D E$ & $M d n$ & $M$ & $D E$ \\
\hline Aporte de resolución de la tarea & .85 & .81 & .13 & .82 & .79 & .17 \\
\hline Afirmación & .82 & .80 & .16 & .82 & .79 & .17 \\
\hline Afirmac. fund. de resol. tarea & .33 & .42 & .29 & .28 & .41 & .41 \\
\hline Afirmac. no fund. de resol. tarea & .79 & .72 & .25 & .61 & .63 & .28 \\
\hline Afirmac. reactiva (feedback) & .66 & .63 & .25 & .43 & .40 & .25 \\
\hline Pregunta & .50 & .49 & .32 & .33 & .32 & .28 \\
\hline Preg. cog. de requer. de opinión/conf. & .60 & .54 & .37 & .33 & .27 & .29 \\
\hline Respuesta & .00 & .27 & .36 & .00 & .18 & .36 \\
\hline Resp. no fund. de resol. tarea & .10 & .26 & .35 & .00 & .18 & .39 \\
\hline Aporte organ. tarea vinculado a su resol. & .65 & .60 & .22 & .44 & .41 & .24 \\
\hline Lectura verbal de consigna/ítems & .62 & .60 & .32 & .21 & .34 & .29 \\
\hline Lectura verbal exploratoria de tabla & .50 & .55 & .31 & .50 & .49 & .36 \\
\hline Orientación & .00 & .22 & .31 & .00 & .10 & .27 \\
\hline Aporte organ. tarea no vinculado a su resol. & .50 & .44 & .33 & .33 & .44 & .33 \\
\hline
\end{tabular}

Nota 1: Valores de intercambio sociocognitivo posibles entre 0 (asimetría extrema) y 1 (simetría extrema).

Nota 2: Los valores de $n$ corresponden a la cantidad de díadas por cada condición. Sin embargo, en relación a cada categoría específica, podía ocurrir que la cantidad de casos válidos difiera respecto a $n$, porque las díadas con ausencia total de dicho código fueron consideradas como casos perdidos. (Las notas 1 y 2 también se aplican a las tablas restantes).

\section{(b) Análisis de la interacción colaborativa en función de la afinidad socioafectiva de la díada}

La Tabla 3 contiene los valores descriptivos de la interacción colaborativa en función de la presencia y ausencia de afinidad socioafectiva recíproca entre los compañeros de díada.

Las unidades de aporte a la resolución de la tarea (nivel 1), así como las subcategorías afirmaciones, preguntas y respuestas (nivel 2), mostraron distribuciones más o menos similares en las díadas con y sin afinidad socioafectiva recíproca, con una leve tendencia de intercambio más equitativo a favor de las primeras. Sin embargo, el análisis más minucioso de los subtipos de afirmaciones de resolución de la tarea de nivel 3 (fundamentadas, no fundamentadas y reactivas) mostró una profundización de la tendencia previa, incluso logrando valores significativos en el caso de las afirmaciones no fundamentadas $(U=163.50 ; p<.05)$.

Por otro lado, en cuanto a las unidades organizativas vinculados a la resolución de la tarea (nivel 1) y sus subcategorías (nivel 2), se observó una tendencia inversa a lo anterior. En ese sentido, los valores de mayor simetría del intercambio se registraron en las díadas sin afinidad socioafectiva recíproca. Lo mismo se observó en cuanto a las unidades organizativas no vinculadas a la resolución de la tarea (nivel 1). 
Tabla 3

Valores descriptivos de cada categoría de la interacción en función de la afinidad socioafectiva de la díada

\begin{tabular}{lcccccc}
\hline & \multicolumn{5}{c}{ Afinidad socioafectiva recíproca } \\
\cline { 2 - 7 } & \multicolumn{3}{c}{ Con afin. $(n=21)$} & \multicolumn{3}{c}{ Sin afin. $(n=24)$} \\
\cline { 2 - 7 } & $M d n$ & $M$ & $D E$ & $M d n$ & $M$ & $D E$ \\
\hline Aporte de resolución de la tarea & .84 & .82 & .10 & .84 & .78 & .17 \\
Afirmación & .84 & .82 & .14 & .78 & .77 & .18 \\
$\quad$ Afirmac. fund. de resol. tarea & .60 & .48 & .36 & .33 & .35 & .32 \\
Afirmac. no fund. de resol. tarea & .87 & .77 & .23 & .64 & .61 & .27 \\
Afirmac. reactiva (feedback) & .57 & .57 & .25 & .45 & .49 & .29 \\
Pregunta & .46 & .43 & .29 & .42 & .39 & .34 \\
$\quad$ Preg. cog. de requer. de opinión/conf. & .50 & .42 & .33 & .33 & .41 & .49 \\
Respuesta & .00 & .21 & .31 & .00 & .24 & .40 \\
$\quad$ Resp. no fund. de resol. tarea & .10 & .22 & .31 & .00 & .23 & .40 \\
Aporte organ. tarea vinculado a su resol. & .45 & .48 & .26 & .54 & .54 & .24 \\
Lectura verbal de consigna/ítems & .35 & .43 & .33 & .56 & .52 & .34 \\
Lectura verbal exploratoria de tabla & .45 & .51 & .30 & .53 & .54 & .36 \\
Orientación & .00 & .18 & .32 & .00 & .16 & .29 \\
Aporte organ. tarea no vinculado a su resol. & .34 & .42 & .30 & .50 & .46 & .35 \\
\hline
\end{tabular}

Nota: $M d n=$ mediana; $M$ = media; $D E$ = desviación estándar.

(c) Análisis de la interacción colaborativa en función de la simetría-asimetría de competencia y de la afinidad socioafectiva de la díada

En este apartado se presentan los resultados del análisis sobre si la simetría-asimetría de competencia interactúa o es regulada por la afinidad socioafectiva entre los compañeros de díada. La tabla 4 presenta los valores descriptivos de cada categoría de interacción en las condiciones de comparación.

En relación a los aportes a la resolución de la tarea (nivel 1), y particularmente las afirmaciones (nivel 2), se registró una mayor tendencia diferenciadora por parte de la afinidad socioafectiva al interior de las díadas con asimetría de competencia, comparado con las díadas simétricas. Esto es, la ausencia de afinidad socioafectiva en las díadas asimétricas tendió a acrecentar la desigualdad del intercambio en este tipo de unidades, con respecto a aquellas que sí contaban con afinidad entre los compañeros. Esta tendencia se acentuó más todavía (alcanzando valores de significación) en categorías de resolución de la tarea de nivel 3: afirmaciones fundamentadas $(U=30.50$; $p<.05)$ y no fundamentadas $(U=28.00 ; p<.05)$. Lo mismo se observó al sumar las afirmaciones y respuestas fundamentadas $(U=28.00 ; p<.05)$. En cambio, en las díadas simétricas, la diferenciación asociada con la presencia o ausencia de afinidad socioafectiva fue muy poco marcada. 
Tabla 4

Valores descriptivos de cada categoría de la interacción, en función de la simetría-asimetría de competencia y de la afinidad socioafectiva de la díada

\begin{tabular}{|c|c|c|c|c|c|c|c|c|c|c|c|c|}
\hline & \multicolumn{6}{|c|}{ Simetría de competencia } & \multicolumn{6}{|c|}{ Asimetría de competencia } \\
\hline & \multicolumn{3}{|c|}{ Con afin. $(n=10)$} & \multicolumn{3}{|c|}{ Sin afin. $(n=13)$} & \multicolumn{3}{|c|}{ Con afin. $(n=11)$} & \multicolumn{3}{|c|}{ Sin afin. $(n=10)$} \\
\hline & $M d n$ & $M$ & $D E$ & $M d n$ & $M$ & $D E$ & $M d n$ & $M$ & $D E$ & $M d n$ & $M$ & $D E$ \\
\hline Aporte de resol tarea & .82 & .79 & .11 & .87 & .83 & .15 & .87 & .86 & .09 & .73 & .73 & .19 \\
\hline Afirmación & .84 & .81 & .17 & .81 & .79 & .15 & .88 & .83 & .10 & .74 & .74 & .20 \\
\hline Afirmac. fund. & .45 & .40 & .33 & .33 & .44 & .27 & .66 & .56 & .38 & .04 & .24 & .36 \\
\hline Afirmac. no fund. & .87 & .80 & .23 & .78 & .70 & .24 & .75 & .74 & .24 & .42 & .50 & .27 \\
\hline Afirmac. reactiva & .71 & .70 & .21 & .66 & .61 & .26 & .50 & .45 & .23 & .25 & .35 & .26 \\
\hline Pregunta & .50 & .47 & .26 & .50 & .46 & .36 & .33 & .39 & .33 & .33 & .32 & .32 \\
\hline Requer. opinión/conf. & .55 & .51 & .35 & .66 & .56 & .40 & .42 & .31 & .27 & .08 & .24 & .32 \\
\hline Respuesta & .10 & .18 & .22 & .25 & .41 & .46 & .00 & .25 & .42 & .00 & .11 & .31 \\
\hline Resp. no fund tarea & .20 & .21 & .21 & .00 & .36 & .48 & .00 & .25 & .50 & .00 & .13 & .33 \\
\hline Aporte org. (resol.) & .68 & .64 & .24 & .64 & .59 & .21 & .33 & .33 & .18 & .48 & .48 & .26 \\
\hline Lectura consigna/ítems & .62 & .62 & .33 & .62 & .58 & .34 & .19 & .25 & .22 & .47 & .45 & .33 \\
\hline Lectura explor. tabla & .48 & .48 & .26 & .64 & .57 & .33 & .43 & .54 & .36 & .50 & .50 & .40 \\
\hline Orientación & .00 & .15 & .27 & .17 & .28 & .34 & .09 & .24 & .39 & .00 & .00 & .00 \\
\hline Aporte org. (no resol.) & .53 & .50 & .33 & .25 & .36 & .31 & .33 & .35 & .27 & .66 & .59 & .38 \\
\hline
\end{tabular}

Nota: $M d n=$ mediana; $M=$ media; $D E$ = desviación estándar.

Respecto a las unidades organizativas vinculadas a la tarea, la afinidad socioafectiva también se asoció con variaciones marcadas al interior de las díadas con asimetría de competencia, pero en un sentido inverso a las afirmaciones cognitivas. En el grupo de asimetría de competencia, la ausencia de afinidad socioafectiva se vinculó con patrones más equitativos de distribución, en comparación con la presencia de afinidad.

Por último, las unidades cognitivas no vinculadas a la resolución de la tarea (nivel 1) también mostraron diferenciaciones aunque en menor grado que las categorías previas, al interior de las condiciones de simetría y asimetría de competencia cognitiva, en función de la afinidad socioafectiva. Así, en las díadas de competencia simétrica la presencia de afinidad entre los compañeros se asoció con intercambios más equitativos; en cambio, en las díadas con asimetría de competencia, la ausencia de afinidad se vinculó con intercambios más homogéneos.

\section{Discusión}

El objetivo de la investigación fue analizar la interacción colaborativa, desde el punto de vista de la distribución de los aportes verbales entre ambos compañeros de trabajo, en función de dos variables psicosociales: la simetría-asimetría de competencia y la afinidad socioafectiva recíproca. Esto se realizó en una tarea de comprensión colaborativa de una tabla de frecuencias en díadas de $6 .^{\circ}$ y $7 .^{\circ}$ grado de primaria. Se compararon díadas simétricas con díadas asimétricas que, a su vez, estaban integradas por individuos con y sin afinidad socioafectiva entre sí. 
El microanálisis de la interacción colaborativa consideró tres tipos generales de unidades cognitivas: de aporte cognitivo a la resolución de la tarea, de organización de la tarea vinculado directamente a su resolución, y de organización de la tarea no vinculado a su resolución. A esto se le sumaron subcategorías más específicas, de nivel 2 y 3 . Todas estas categorías y subcategorías verbales fueron estudiadas en función de la simetría-asimetría de competencia, de la afinidad socioafectiva y de la combinación de ambas variables, acorde a los tres objetivos específicos inicialmente planteados.

En relación al primer objetivo específico, analizar cada categoría de interacción en función de la simetríaasimetría de competencia, los resultados generales son coincidentes con los reportados en trabajos previos (Castellaro \& Roselli, 2018b; Schmitz \& Winskel, 2008). En tal sentido, partiendo de la estrecha conexión entre cognición, interacción y tarea, se corrobora que la similitud o diferencia entre las habilidades de los compañeros se traducen en formas sociocognitivas particulares (Asterhan et al., 2014; Buchs \& Butera, 2009; Buchs et al., 2008; Denessen et al, 2008). Esto es, la igualdad en los niveles de competencia entre los compañeros de trabajo tiende a asociarse con aportes comunicacionales más equitativos, especialmente en las unidades vinculadas a la resolución de la tarea y su regulación. Por contrapartida, la desigualdad cognitiva tiende a expresarse en términos de asimetría en las contribuciones de los participantes durante la interacción sociocognitiva.

Dentro de las investigaciones sobre interacciones sociocognitivas infantiles, esta hipótesis fue considerada fundamentalmente en tareas de resolución de problemas lógicos (Asterhan et al., 2014; Castellaro \& Roselli, 2018b; Wegerif et al., 2016; Yang, 2015) o de construcción de nociones primarias del pensamiento en el sentido piagetiano del término (Zapiti \& Psaltis, 2012, 2019). Tradicionalmente, ambos tipos de tareas fueron vinculadas con la construcción de organizadores formales o sintácticos del pensamiento, relativamente independientes de dominios específicos. El caso puntual de la comprensión de tablas parece diferenciarse en este sentido. Martí et al. (2011) plantea que, desde una perspectiva piagetiana clásica, sería esperable que los estudiantes de $6 .^{\circ}$ y $7 .^{\circ}$ grado sean capaces de organizar la información presentada, puesto que se trataría de una capacidad adquirida alrededor de los ocho años cuando se ingresa al estadio operatorio concreto, en tanto la comprensión de una tabla requiere de asociaciones entre dos variables. Sin embargo, tal como lo muestran sus propios hallazgos, como las reportados en este estudio, una parte importante de los estudiantes de esas edades solo ha alcanzado un nivel básico de comprensión de la tabla, lo cual estaría sugiriendo que se trata de un proceso que requiere el desarrollo de elementos específicos complementarios (Curcio, Peralta, \& Castellaro, 2019). En tal sentido, el sistema educativo formal cumple un papel importante en tanto considera, entre sus contenidos curriculares, aquellos relativos a la comprensión, construcción y uso de este tipo de sistemas, como son las tablas y los gráficos.

El segundo objetivo específico fue analizar la interacción colaborativa en función de la presencia o ausencia de afinidad socioafectiva recíproca entre los compañeros de la díada. Las unidades de resolución de la tarea, específicamente diferenciadas entre afirmaciones fundamentadas, no fundamentadas y reactivas, se distribuyeron más equitativamente en las díadas con afinidad socioafectiva, en comparación con las díadas sin dicha afinidad. Estudios previos (McDonald et al., 2002; Strough et al., 2001) ya habían sugerido una relación positiva entre la afinidad, por un lado, y un mayor volumen y calidad en la comunicación verbal de la díada, por el otro. Pero estas conclusiones estaban referidas a tareas libres 0 expresivas. Aquí se analizó una actividad diferente (comprensión de tablas de frecuencias) y el foco estuvo puesto en el grado de distribución de los aportes individuales durante la interacción (y no tanto en el volumen comunicacional en su conjunto). A pesar de esto, puede afirmarse que los hallazgos son 
complementarios y condicen con los reportados en dichos antecedentes.

En cambio, en relación a las unidades organizativas de la tarea, ya sean vinculadas o no a su resolución, se observó una tendencia contraria a la anterior. Así, ambas se distribuyeron más equitativamente en las díadas sin afinidad socioafectiva, en comparación con las díadas con afinidad. Una explicación plausible de esto es que las unidades cognitivas organizativas dependen más de una impronta individual que las referidas a los aportes cognitivos. En estos últimos, el compartir contribuciones cognitivas que hacen a la resolución de la tarea resulta facilitado con alguien con el que se tiene afinidad socioafectiva; no ocurriría lo mismo con los aportes organizativos que, como se dijo, son más personales y, por lo tanto, estarían menos influenciados por dicha afinidad.

Desde un punto de vista conceptual, el concepto de afinidad socioafectiva se asocia con la noción de sentimientos interindividuales propuesta por Piaget (1995), que se traduce en la polaridad simpatía y antipatía. Según el autor, los sentimientos de simpatía surgen hacia las personas que comparten los intereses del sujeto y que lo valorizan como persona. Esto implica un aprecio mutuo entre las partes, por un lado, y una escala común de valores que favorecen el intercambio, por el otro. Por contrapartida, la antipatía implica la ausencia de los aspectos anteriores. El surgimiento evolutivo de los sentimientos interindividuales coincide con la primera infancia, aunque estos se complejizan a medida que avanza en el desarrollo. Los primeros sentimientos morales están asociados con el respeto unilateral hacia una figura superior adulta, pero en la segunda infancia aparece un nuevo sentimiento asociado con la cooperación entre pares: el respeto mutuo. Esto implica una valoración recíproca similar entre los compañeros, lo cual generalmente forma parte de la amistad fundada en la estimación interpersonal bilateral. Lo anterior condice con la relación teórica entre la afinidad socioafectiva de los sujetos y la propia interacción social entre estos, tal como ya sugieren los antecedentes mencionados.
El tercer objetivo específico constituyó el propósito más importante del trabajo. El aporte principal de la investigación fue proponer un enfoque psicosocial de la interacción colaborativa y, en ese sentido, la igualdad o diferencia entre las competencias específicas fue estudiada de manera integrada a una variable psicosocial, que en este caso fue la afinidad socioafectiva entre los individuos. El principal resultado estuvo referido al papel modulador diferencial de la afinidad socioafectiva, según se trate de la simetría o asimetría entre las competencias de los compañeros de díada. En este sentido, en la condición de asimetría de competencia, la afinidad socioafectiva tiende a reducir el desequilibrio entre los aportes de los compañeros de trabajo, específicamente en lo concerniente a las afirmaciones fundamentadas y no fundamentadas. En cambio, en la simetría de competencia, la afinidad socioafectiva prácticamente no generó diferenciaciones en general.

Estos resultados son coherentes con una perspectiva psicosocial de la interacción sociocognitiva, revalorizando a esta última como un proceso de influencia social que transciende los elementos puramente cognitivos o epistémicos implicados (Castorina, 2018; Quiamzade et al., 2014; Staerklé \& Butera, 2017). Por consiguiente, las igualdades o diferencias interpersonales devienen, respectivamente, en fuentes de simetría o asimetría psicosocial, incluso los aspectos pretendidamente más «objetivos» como puede ser el nivel de competencia específica sobre la tarea. Por lo tanto, en el caso puntual de la asimetría de competencia, que potencialmente constituye una fuente inicial de influencia asimétrica psicosocial, la ausencia de afinidad socioafectiva entre los compañeros agrega un segundo elemento de desequilibrio. En cambio, la presencia de afinidad socioafectiva tendería a reducir la brecha en la contribución comunicacional cognitiva entre los compañeros, especialmente en lo que hace a los aportes fundamentados y no fundamentados relativos a la resolución de la tarea, lo cual explica la importancia de esta variable como reguladora del mayor o menor equilibrio interactivo. 
Ahora bien, en el caso de las díadas con simetría de competencia y, por consiguiente, potencialmente simétricas en términos de influencia social, la presencia o ausencia de afinidad socioafectiva tiende asociarse únicamente con aspectos puntuales, léase, aquellos no vinculados con la resolución de la tarea. Posiblemente, esto se explique por el hecho de que las díadas simétricas estaban integradas por estudiantes de baja competencia: en tanto estos poseen menos habilidades para enfrentar cognitivamente la tarea, es esperable que la interacción se dirima sobre todo a nivel de los aspectos no vinculados con su resolución. En tal sentido, sería interesante plantear nuevos análisis en los cuales se compare lo anterior con otras díadas simétricas, pero integradas por compañeros de alta competencia y, en este caso, observar si la afinidad socioafectiva tiende a generar diferenciaciones en las categorías vinculadas y no vinculadas con la resolución de la tarea.

Por último, no debe olvidarse el nivel de exigencia cognitiva, en términos de competencia individual, que demanda la resolución de una tarea de comprensión de tablas de frecuencias. Por consiguiente, cuando eso es trasladado a un marco de resolución colaborativa, por ejemplo en díadas, la igualdad o diferencia entre los niveles individuales de los estudiantes constituye un regulador primario de la interacción sociocognitiva. Sin embargo, al mismo tiempo, esta sufre diferentes variaciones al combinarse con los demás aspectos psicosociales involucrados en la situación social, entre los cuales se encuentra la afinidad socioafectiva. En el caso de la simetría de competencia, esta de por sí tiende a asegurar una base de intercambio equitativo que no sufre variaciones significativas en función de la afinidad socioafectiva; excepto en aquellos aspectos más ajenos a la estricta resolución de la tarea. En cambio, en el caso de la asimetría de competencia, la desigualdad en los aportes individuales orientados a la resolución u organización de la tarea sí puede ser matizada por la presencia de afinidad socioafectiva. En el caso puntual de la asimetría de competencia, si bien en este estudio no se analizaron modalidades o cualidades interactivas, podría hipotetizarse una asociación entre la presencia de afinidad socioafectiva y una modalidad sociocognitiva con rasgos de tutoría o andamiaje; o, por el contrario, una asociación entre la ausencia de afinidad sociofectiva y una modalidad sociocognitiva con rasgos de imposición unilateral por parte del estudiante de mayor competencia. Estudios anteriores que han estudiado situaciones de asimetría de competencia han concluido similarmente, aunque en relación a otra variable psicosocial como es el género (Leman \& Duveen, 1999; Psaltis \& Duveen, 2007; Zapiti \& Psaltis, 2012, 2019), lo cual aporta un elemento complementario de apoyo a dicha hipótesis.

La conclusión más general que puede extraerse es que el conjunto de resultados pone de relieve la importancia de tener un enfoque psicosocial, concreto y situado de los miembros de la díada en interacción. Esto abre las puertas a la consideración de las múltiples variables psicosociales y contextuales intervinientes en la interacción sociocognitiva colaborativa, además del aspecto puramente cognitivo. El presente estudio se limitó a efectuar un microanálisis de la interacción colaborativa en relación a las dos variables independientes mencionadas. En este sentido, la finalidad fue básicamente descriptiva. Queda pendiente para futuras investigaciones el análisis de los pre-tests en relación a la performance colectiva, y los posibles efectos a aprendizaje que pueden lograr individualmente a partir de los distintos tipos de interacción.

\section{Conflicto de intereses}

Los autores declaran que el presente manuscrito no presenta conflicto de interés alguno (moral, económico, laboral o de investigación).

\section{Responsabilidad ética}

Este trabajo se realizó siguiendo los lineamientos éticos y demás regulaciones nacionales e internacionales correspondientes. Se obtuvieron los consentimientos informados de los participantes. No 
participaron del estudio quiénes no contaron con dicha autorización. En ninguna parte del trabajo aparecen datos que permitan la identificación de los participantes. El proyecto de base estuvo aprobado por el Consejo de Ética y Derechos Humanos del Ministerio de Educación de Santa Fe (CEDEHME) (sin número de autorización).

\section{Contribución de autoría}

MC: concepción y diseño del estudio, interpretación de los datos, escritura y revisión final del manuscrito.

NR: concepción y diseño del estudio, interpretación de los datos, escritura y revisión final del manuscrito.

\section{Agradecimientos}

A los diferentes actores de las comunidades educativas (directivos, docentes, personal no docente, adultos responsables de los alumnos, etc.) y, en especial, a los propios participantes. Asimismo, un profundo agradecimiento a la Dra. Nadia Peralta y a los estudiantes universitarios Juan Manuel Curcio, Romina Leguizamón, Nicolás Morguen y Macarena Rondini, por su colaboración indispensable en el trabajo de campo.

\section{Referencias}

Andersen, C., Scheuer, N., Pérez, M., \& Teubal, E. (2017). Representational Systems and Practices as Learning Tools. Neterhlands: Sense Publishers. doi: $10.1163 / 9789087905286$

Asterhan, C., Schwarz, B., \& Cohen-Eliyahu, N. (2014). Outcome feedback During Collaborative Learning: Contingencies Between Feedback and Dyad Composition. Learning and Instruction, 34, 1-10. doi: 10.1016/j.learninstruc.2014.07.003

Buchs, C., \& Butera, F. (2009). Is a Partner's Competence Threatening During Dyadic Cooperative Work? It Depends on Resource Interdependence. European Journal of Psychology of Education, 24(2), 145-154. doi: 10.1007/BF03173007
Buchs, C., Darnon, C., Quiamzade, A., Mugny, G., \& Butera, F. (2008). Conflits et apprentissage. Régulation des conflits sociocognitifs et apprentissage. Revue Française de pédagogie, 163, 105-125. doi: 10.4000/rfp.1013

Castellaro, M., \& Roselli, N. (2018a). Interacción sociocognitiva entre pares en situaciones simétricas y asimétricas de competencia epistémica. Revista de Psicología, 36(1), 333-365. doi: 10.18800/psico.2 01801.011

Castellaro, M., \& Roselli, N. (2018b). Resolución colaborativa de problemas lógicos en condiciones de simetría y asimetría cognitiva. Revista Propósitos y Representaciones, 6(1), 135-166. doi: 10.20511/ pyr2018.v6n1.196

Castorina, J. A. (2018). Psicología genética y psicología social: ¿Dos caras de una misma disciplina o dos programas de investigaciones compatibles? In A. Barreiro (Comp.), Representaciones Sociales, Prejuicio y Relaciones con los Otros. La Construcción del Conocimiento Social y Moral (pp. 33-53). Buenos Aires: UNIPE Editorial Universitaria.

Curcio, J. M., Peralta, N. S., \& Castellaro, M. (2019). Tamaño del grupo, argumentación y lectura de tablas en estudiantes universitarios. Diversitas, Perspectivas en Psicología. 15(2). doi: 10.15332/ 22563067.4350

Denessen, E., Veenman, S., Dobbelsteen, J., \& Schilt, J. van (2008). Dyad Composition Effects on Cognitive Elaboration and Student Achievement. Journal of Experimental Education, 76(4), 363-383. doi: 10.32 00/JEXE.76.4.363-386

Gabucio, F., Martí, E., Enfedaque, J., Gilabert, S., \& Konstantinidou, A. (2010). Niveles de comprensión de las tablas en alumnos de primaria y secundaria. Cultura y Educación, 22(2), 183-197. doi: 10.1174/ 113564010791304528

Leman, P., \& Duveen, G, (1999). Representations of Authority and Children's Moral Reasoning. European Journal of Social Psychology, 29(5-6), 557-575. doi: 10.1002/(SICI)1099-0992(199908/09)29:5/ 6<557::AID-EJSP946>3.0.CO;2-T 
Maita, M., Mareovich F., \& Peralta, O. (2014). Intentional teaching Facilitates Young Children's Comprehension and Use of a Symbolic Object. Journal of Genetic Psychology, 175(5), 401-415. doi: 10.1080/002213 25.2014.941320

Martí, E., García-Milá, M., Gabucio, F., \& Konstantinidou, K. (2011). The Construction of a Double-Entry Table: A Study of Primary and Secondary School's Students Difficulties. European Journal of Psychology of Education, 26, 215-234. doi: 10.1007/s10212-010-0046-1

Martí, E., Scheuer, N., Cavalcante, S., Trench, M., \& Brizuela, B. M. (2016). Symbolic Representation of the Number Three: A Study with Three-Year-Old Children from Contrasting Socioeconomic Environments. Journal of Cognitive Psychology, 28(6), 743-755. doi: 10.1080/20445911.2016.11 88821

McDonald, R., Miell, D., \& Mitchell, L. (2002). An Investigation of Children's Musical Collaboration: The Effects of Friendship and Age. Psychology of Music, 30(2), 148-163. doi: 10.1177/030573560 2302002

Peralta, N., \& Roselli, N. (2017). Modalidad argumentativa en función del tipo de tarea y tamaño del grupo. Cogency. Journal of Reasoning and Argumentation, 9(2), 67-83. Recuperado de http:// cogency.udp.cl/index.php/cogency/article/view/295

Pérez, M., Martí, E., \& Pozo, J. (2010). Los sistemas externos de representación como herramientas de la mente. Cultura y Educación, 22(2), 133-147. doi: $10.1174 / 113564010791304519$

Pérez, M., Postigo, Y., \& Marín, C. (2018). Understanding of Graphs in social Science Undergraduate Students: Selection and Interpretation of Graphs. Irish Educational Studies, 37(3), 1-23. doi: 10.1080/0332 3315.2018.1440248

Piaget, J. (1984). El Criterio Moral en el Niño. Barcelona: Martínez Roca (Trabajo original publicado en 1932).

Piaget, J. (1995). Seis Estudios de Psicología. Barcelona: Labor (Trabajo original publicado en 1964).
Postigo, Y., \& López-Manjón, A. (2015). Alfabetización gráfica en libros de texto de biología: análisis de las actividades con imágenes. Infancia y Aprendizaje, 38(3), 509-541. doi: 10.1080/02103702.2015.1054667

Pozo, J. I. (2017). Aprender más allá del cuerpo: de las representaciones encarnadas a la explicitación mediada por representaciones externas. Infancia $y$ Aprendizaje, 40(2), 219-276. doi: 10.1080/021037 02.2017.1306942

Psaltis, C. (2011). The Constructive Role of Gender Asymmetry in Social Interaction: Further Evidence. British Journal of Developmental Psychology, 29(2), 305-312. doi: 10.1111/j.2044-835X.20 11.02029.x

Psaltis, C., \& Duveen, G. (2007). Conservation and Conversation Types: Forms of Recognition and Cognitive Development. British Journal of Developmental Psychology, 25(1), 79-102. doi: 10.1 348/026151005X91415

Psaltis, C., Duveen, G., \& Perret-Clermont, A. (2009). The Social and the Psychological: Structure and Context in Intellectual Development. Human Development, 52(5), 291-312. doi: 10.1159/ 000233261

Psaltis, C., \& Zapiti, C. (2014). Interaction, Communication and Development: Psychological Development as a Social Process. Reino Unido: Routledge.

Quiamzade, A., Mugny, G., \& Butera, F. (2014). Psychologie sociale de la connaissance. Grenoble, Francia: PUG.

Rodríguez, J., Martí, E., \& Salsa, A. (2018). Symbolic Representations and Cardinal Knowledge in 3- and 4Year-Old Children. Cognitive Development, 48, 235243. doi: 10.1016/j.cogdev.2018.09.004

Roselli, N. (2011). Proceso de construcción colaborativa a través del chat según el tipo de tarea. Revista de Psicología, 29(1), 3-36. Recuperado de http:// revistas.pucp.edu.pe/index.php/psicologia/article/ view/1142

Roselli, N. (2016). Modalities to Collaborate in the Social Construction of Conceptual Maps: A Comparison between Individual and Collective Productions. 
American Journal of Educational Research, 5(10), 1058-1064. doi: 10.12691/education-5-10-7

Roselli, N., Dominino, M., \& Peralta, N. S. (2010). Influencia del tipo de tarea sobre la interacción colaborativa en equipos virtuales. Revista de Psicología General y Aplicada, 63(1-2), 97-117.

Salsa, A., \& Vivaldi, R. (2017). Developmental Changes in Early Comprehension and Production of Drawings: Evidence from Two Socioeconomic Backgrounds. Journal of Genetic Psychology, 178(4), 217-228. doi: 10.1080/00221325.2017.1328385

Salsa, A., \& Gariboldi, M. B. (2017). Experiencia con símbolos y comprensión de dibujos en niños pequeños de distintos contextos socioeconómicos. Avances en Psicología Latinoamericana, 36(1), 2943. doi: 10.12804/revistas.urosario.edu.co/apl/a.4332

Schmitz, M., \& Winskel, H. (2008). Towards Effective Partnerships in a Collaborative Problem-Solving Task. British Journal of Educational Psychology, 78(4), 581-596. doi: 10.1348/000709908X281619

Staerklé, C., \& Butera, F. (2017). Conflits Constructifs, Conflicts Destructifs. Regards Psychosociaux. Lausana, Suiza: Antípodes.

Strough, J., Berg, C., \& Meegan, S. (2001). Friendship and Gender Differences in Task and Social Interpretations of Peer Collaborative Problem Solving. Social Development, 10(1), 1-22. doi: 10.11 11/1467-9507.00145
Strough, J., Swenson, L., \& Cheng, S. (2001). Friendship, Gender and Preadolescents' Representations of Peer Collaboration. Merril-Palmer Quarterly, 47(4), 475499. doi: $10.1353 / \mathrm{mpq} .2001 .0025$

Swenson, L., \& Strough, J. (2008). Adolescents' Collaboration in the Classroom: Do Peer Relationships or Gender Matter? Psychology in the Schools, 45(8), 715-728. doi: 10.1002/pits.20337

Wegerif, R., Fujita, T., Doney, J., Pérez-Linares, J., Richards, A., \& Rhyn, C. van (2016). Developing and Trialing a Measure of Group Thinking. Learning and Instruction, 48, 40-50. doi: 10.1016/j.learninstru c.2016.08.001

Yang, Y. (2015). Lessons Learnt from Contextualizing a UK Teaching Thinking Program in a Conventional Chinese Classroom. Thinking Skills and Creativity, 19, 198-209. doi: 10.1016/j.tsc.2015.07.002

Zapiti, A., \& Psaltis, C. (2012). Asymmetries in Peer Interaction: The Effect of Social Representations of Gender and Knowledge Asymmetry on Children's Cognitive Development. European Journal of Social Psychology, 42(5), 578-588. doi: 10.1002/ejsp.1885

Zapiti, A., \& Psaltis, C. (2019). Too Good to Be True? Towards an Understanding of the Zone of Proximal Development (ZPD) Dynamics from a Piagetian Perspective: Gender Composition and its Changing Role from Early to Middle Childhood. Psihologija, 52(4), 323-345. doi: 10.2298/PSI181023006Z

\section{Mariano Castellaro}

Consejo Nacional de Investigaciones Científicas y Técnicas, Argentina. Universidad Nacional de Rosario, Argentina

Doctor en Psicología. Cuenta con investigaciones sobre interacción colaborativa infantil, aprendizaje colaborativo y educación. Su línea de investigación refiere a los procesos sociales y cognitivos implicados en el desarrollo cognitivo infantil y la construcción de conocimientos escolares.

ORCID: 0000-0001-5470-9662

Autor corresponsal: castellaro@irice-conicet.gov.ar

\section{Néstor Roselli}

Centro de Investigaciones en Psicología, Pontificia Universidad Católica Argentina. Consejo Nacional de Investigaciones Científicas y Técnicas, Argentina

Doctor en Psicología. Cuenta con investigaciones sobre interacción colaborativa, aprendizaje colaborativo y educación. Su línea de investigación refiere a los procesos sociales y cognitivos implicados en el desarrollo cognitivo y la construcción de conocimientos en entornos formales.

ORCID: 0000-0002-7313-4566

roselli@irice-conicet.gov.ar 


\section{Anexo}

Tabla utilizada en la tarea de comprensión y primeras preguntas del cuestionario.

\begin{tabular}{|lcccc|}
\hline & Menos de 25 kg & De 25 a 34 kg & De 35 a 44 kg & Más de 44 kg \\
\hline Niños & 1 & 10 & 12 & 2 \\
\hline Niñas & 0 & 6 & 13 & 6 \\
\hline Totales & 1 & 16 & 25 & 8 \\
\hline
\end{tabular}

1. Los números que aparecen en la parte sombreada de la tabla (por ejemplo, $1,10,12$, etc.) se refieren a:
a) Pesos (en kg)
b) Número de personas
c) Edades
d) Alturas

2. Lo que aparece en la parte de arriba de la tabla (por ejemplo, menos de 25, de 25 a 34, de 35 a 44, más de 44) se refiere a:
a) Número de niños y niñas que participaron en el estudio
b) Pesos (en kg)
c) Edades
d) Alturas

3. Los totales se refieren a:
a) niños y niñas
b) niñas
c) niños
d) peso total de niños y niñas

4. En una casilla aparece un 0 , eso quiere decir que:
a) No hay ninguna niña que pese menos de $25 \mathrm{~kg}$
b) No hay ningún niño que pese menos de $20 \mathrm{~kg}$
c) Hay alguna niña que pesa $0 \mathrm{~kg}$
d) No hay ninguna niña que pese más de 44 kg 